\title{
EFFICIENCY ALTERATION OF FRANCIS TURBINES BY TRAVELLING BUBBLE CAVITATION
}

\author{
Experimental and theoretical study
}

\author{
CH. ARN, PH. DUPONT AND F. AVELLAN \\ IMHEF-EPFL \\ 33, av. de Cour, CH-1007 Lausanne, Switzerland \\ Email: christophe.arn@imhef.dgm.epfl.ch
}

\begin{abstract}
.
The setting level of a hydraulic machine, specially for low head machines, is decided with respect to the possible alteration of the efficiency due to the cavity development. This alteration can easily be noticed by following the evolution of the efficiency $\eta$ as a function of the Thoma number $\sigma$ leading to the so-called $\eta-\sigma$ cavitation curves. Observation of the cavity extent in the flow passage of the runner allows to associate the drop of efficiency with a particular type of cavity development.

However, depending on the type of cavities this drop cannot be very easily explained. Obviously, for a leading edge attached cavity corresponding to high head operating points, the presence of the vapour phase on the blade suction side limits the pressure at the vapour tensile strength value which causes the flow alteration. In the case of travelling bubble cavitation, corresponding to the outlet cavitation at the nominal head, previous experiments with a 2-D NACA profile show that the modification of the mean pressure field is mainly due to the bubble dynamics. The aim of this paper is to present the results and the analysis of two experiments intending to explain the influence of the nuclei content on the mean pressure field correction due to the bubble dynamics.
\end{abstract}

\section{Introduction}

Travelling bubble cavitation takes place for the design value of the head, at the throat of the Francis runner flow passage, close to the outlet and corresponds to a low flow angle of attack. The development of this type of cavitation, responsible of an efficiency alteration of the machine is very sensitive to the content of cavitation nuclei and to the value of the setting level. For this reason, this setting level is determined with respect to this type of cavitation. The physical phenomenon underlying such an efficiency 
alteration of the turbine is not yet very clear. Previous experiments [9] performed with a NACA009 profile mounted on a hydrodynamic balance show us the hydrodynamic loads to be dependent on the cavitation coefficient $\sigma$ and on the content of the cavitation nuclei. We can so deduct that these two parameters are responsible for that pressure modification on the blade as the pressure distribution is generating the lift.

An approach to study this influence is to consider that the pressure field modification is mainly due to the bubble dynamics [8] [10] [7]. If we consider the travelling bubble like a non moving sphere in expansion in a still fluid, the computation of the pressure field around the bubble is possible. Indeed, the relative velocity between the bubble and the fluid is very low. The potential theory of incompressible flows allows to class this case as the determination of the pressure generated by a potential $\phi=-\dot{R} R^{2} r^{-1}$ where $R$ is the radius of the sphere. The expansion of this sphere generates a radial pressure field around. The Bernoulli equation leads to the expression of the pressure field $p$. Thus, the pressure on the blade can be obtained by superposing the two bubble potential fields according to the image superposition technique, the wall being a flow surface. The Rayleigh-Plesset model allows us to determine the evolution of the bubbles radius, which is necessary to compute the radial pressure field. In the case of the NACA 009 bidimensional blade whose pressure field is computed by a Navier-Stokes finite elements code, the evolution of the bubbles along streamlines is determined solving the Rayleigh-Plesset equation using a fifth order Runge-Kutta method. One can see an example on Figure 1 with a value of the cavitation coefficient $\sigma$ equal to 0.43 . The evolution of the pressure computed in a point of the wall is also reported.
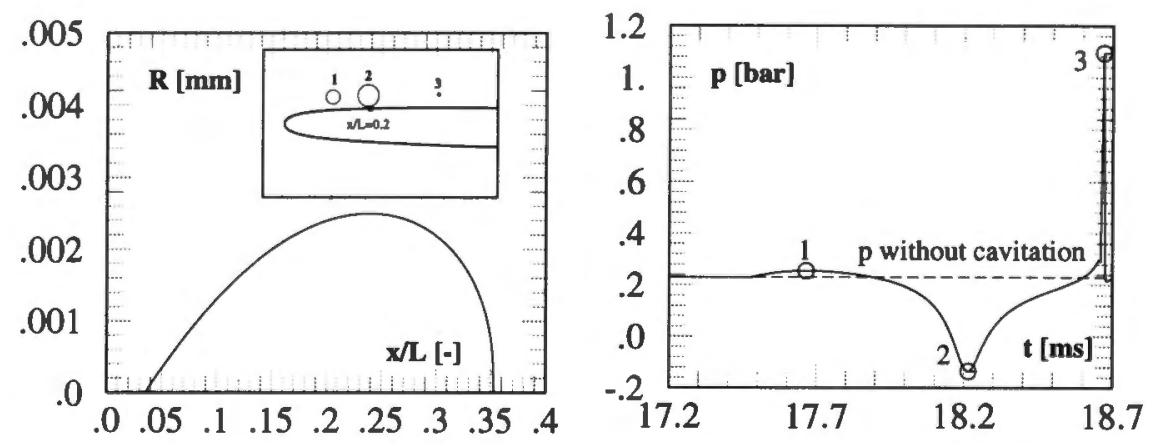

Figure 1. Bubble radius evolution along a streamline in a NACA009 blade and pressure generated at $x / L=0.2$. $\sigma=0.43$.

Obviously, this method is valid for the case where the bubbles remain relatively spherical which corresponds to high values of the cavitation coefficient $\sigma$. Moreover, the amplitude of the pressure are higher as the measured one [3]. Thus, this model allows qualitatively a good approach of the physical phenomenon. For lower values of $\sigma$, the size of the bubbles is greater 
than the layer containing the activated nuclei. Thus, the shape of the bubbles have a trend to be flattened up to form an hemisphere [1]. This trend depends strongly to the Weber number. In this case, the pressure on the wall under the bubble is naturally the vapour pressure value $p_{v}$. If the nuclei content is great enough to obtain the cavitation saturation, the pressure becomes constant at the value of $p_{v}$. Then we can define three different zones of travelling bubble cavitation influence. The first one is the part of the blade where the bubbles are spherical. The pressure modification is there mainly due to the bubble dynamics. The second zone is the region where the bubbles becomes hemispherical and the pressure under the bubbles are limited by the vapour tensile strength. The last one is the part of the blade where a saturation of the development of the bubbles is obtained and where the pressure on the blade is constant and equal to the value of $p_{v}$. The aim of this paper is then to present the results of an experiment intending to confirm these different points by measuring the pressure distribution on a two dimensional blade and on a Francis runner with travelling bubble cavitation.

\section{Experimental set-up}

\subsection{MEASUREMENTS ON THE NACA 009 BLADE}

The test are carried out in the IMHEF high speed cavitation tunnel [5]. The experimental hydro-foil is a 2D NACA $009,100 \mathrm{~mm}$ long and $150 \mathrm{~mm}$ wide, truncated at $90 \%$ of its chord length. 18 piezoresistive absolute pressure transducers are distributed on the suction side of the blade, as shown on Figure 2. The measurement range covers 0 to $100 \mathrm{bar}$. Each transducer is supplied by an independent current source and its output pressure signal is separately amplified and band-pass filtered. Data acquisition is performed with the help of two digital transient recorders with a 12 bits resolution per sample. The first one (Lecroy 8212a) allows simultaneous data acquisition of 32 signals at a maximum sampling frequency of $5 \mathrm{kHz}$ whereas the second one ensures simultaneous data acquisition of 12 channels at a maximum sampling rate of $1 \mathrm{MHz}$ (3 Lecroy 6810 Modules) the static calibration of the pressure transducers is performed with the blade mounted in the test section by varying the static pressure in the tunnel from 0.3 to 10 bar. To achieve a dynamic calibration of the pressure transducers, a special technique is developed to generate a pressure impulse in the test section [6]. The transducers output are compared to the output of a $601 \mathrm{Kistler}$ pressure transducer mounted in the test section too. The main result is a good concordance up to $15-20 \mathrm{kHz}$.

A control of the nuclei content is performed during all the experiment. Indeed, the travelling bubble cavitation is impossible without the injection of cavitation nuclei. The nuclei are generated by an expansion of airsaturated water in a series of injection modules [2]. By varying the number of these modules, one can obtain the required quantity of cavitation nuclei 

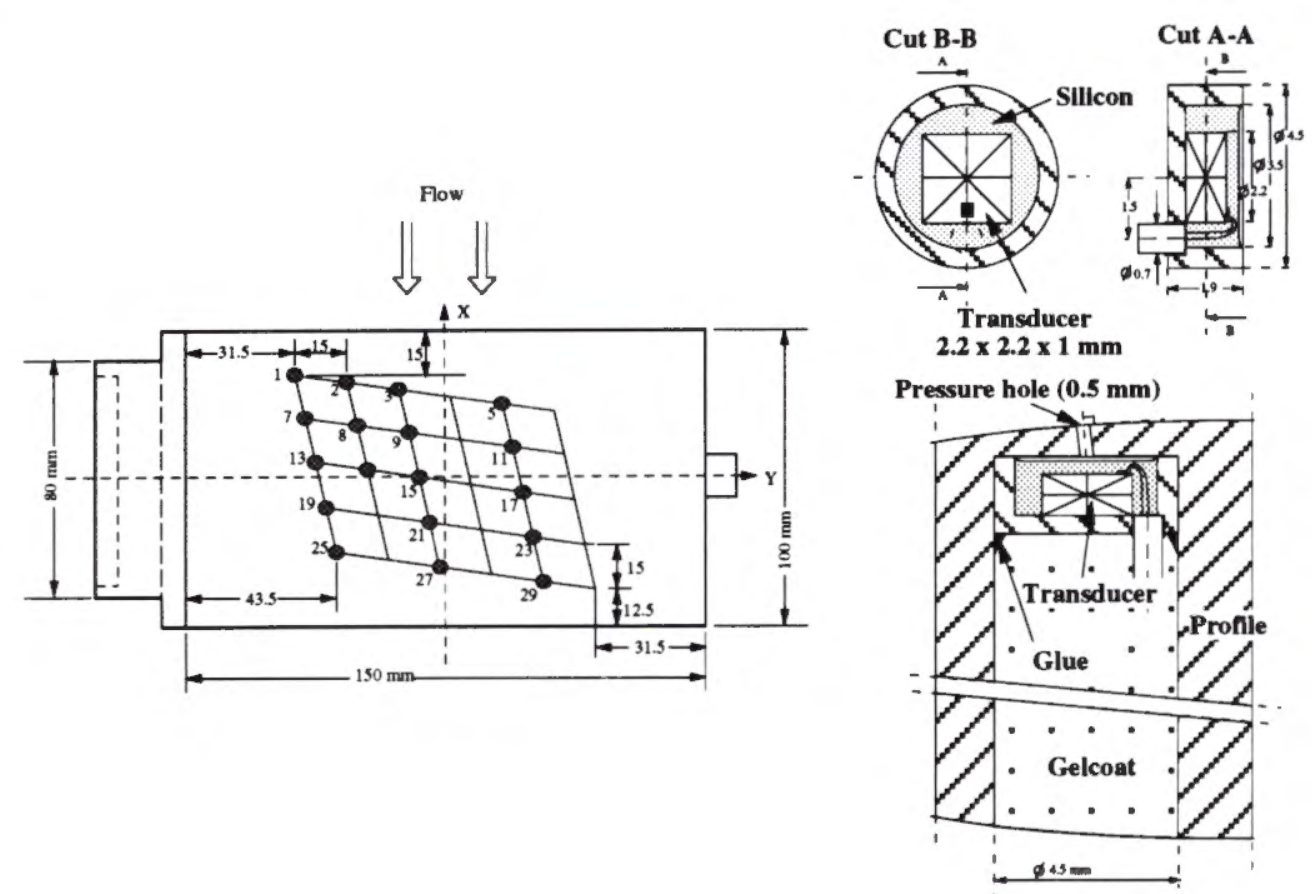

Figure 2. NACA 009 hydro-foil equipped with 15 transient pressure transducers. Pressure transducers mounting

in the test section. The distribution of these nuclei is measured by a cavitation nuclei counter [4] which use the Venturi effect for the detection of the nuclei explosive growth.

\subsection{MEASUREMENTS ON THE FRANCIS RUNNER}

The experiment is conducted with a Francis runner model which has a specific speed $\nu=0.33$. It represents a standard case of a Francis turbine design. The tests of the model are performed on the high performance research test rig of IMHEF. This closed loop test rig covers a range of flow rates up to 1.5 cubic meters per second, with a maximum net head of 60 meters. The primary quantities as the torque, the flow rate, the head, the angular velocity and the water temperature are continuously recorded to determine the power and the efficiency of the machine with an overall accuracy better than 0.1 percent.

Two blades of the runner are equipped with each five absolute transient pressure transducers similar to these mounted on the $2 \mathrm{D}$ blade. The position of the transducers is described on Figure 3. The acquisition set-up is the same as the other experiment except the conditioning electronics connected to the on-board transducers. This electronics is placed in the head of the runner, it has an amplifier with a remotely variable gain and a multiplexer that allows the scanning of all the transducers. Signals are converted to frequency to avoid electro-magnetic disturbances. They are brought out from the rotating part through a slip ring collector and finally reverted to voltage signals which are digitized on the waveform recorders. 


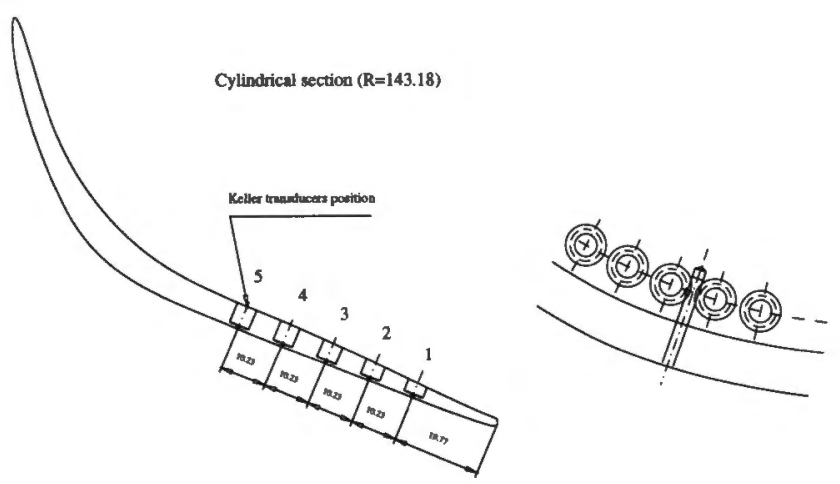

Figure 3. Transducers position on the two blades of the Francis runner

\section{Results and discussion}

\subsection{NACA 009 BLADE}

For the case of the 2D blade, the cavitation coefficient $\sigma=\frac{p_{r e f}-p_{v}}{1 / 2 \rho C^{2}}$ is defined by a relation similar as the definition of the local cavitation factor $\chi_{E}=\frac{p_{r e f}-p_{v}}{\rho E}$ for the turbomachines where $C$ is the velocity and $E$ the energy. A previous experiment conducted with the same blade mounted on a five components hydrodynamic balance give us the evolution of the lift coefficient $c_{z}$ as a function of this cavitation coefficient $\sigma$ [3]. We can observe an increase of the lift from the inception of the travelling bubble cavitation. Then, after a maximum value corresponding to $\sigma=0.32$, the lift falls. These results are reported on the Figure 4.

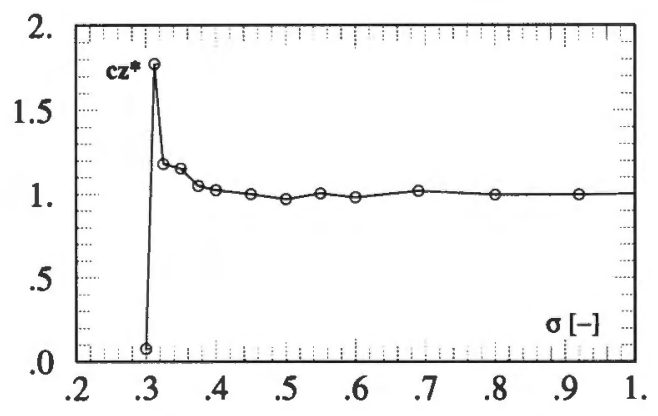

Figure 4. Measured lift coefficient as a function of the cavitation coefficient $\sigma$.

An approach to explain this lift drop phenomenon is to measure the pressure distribution at the same operating points, for an upstream velocity of $20 \mathrm{~m} / \mathrm{s}$ and with cavitation nuclei injection in order to obtain the saturation condition. These distributions are reported on Figure 5 and 
compared with the result of a Navier-Stokes computation. The frames corresponding to each $\sigma$ are too illustrated on this Figure. One can observe a good agreement between the pressure distribution measured for a high value of $\sigma$ and the computed distribution. This provides a certain confidence in the results. From the inception of travelling bubble cavitation, a depression appears in the zone where the size of the bubbles is maximum ( $\sigma=0.4$ and $x / L=0.3$ ). In this condition, the bubbles stay relatively spherical. For a value of $\sigma=0.375$, the bubbles become hemispherical and the depression extends over the suction side but doesn't go below the vapour pressure value. For lower values of $\sigma$, the measured pressures correspond in all positions to the vapour pressure. Indeed, the travelling bubble cavitation saturation is reached as shown in the different frames on Figure 5. The main noticing is that the maximum lift corresponds to the situation where the value of $-\sigma$ reaches the plateau of the pressure coefficient distribution. In fact, this is mainly the position of the point where the pressure is minimum which determine the behavior of the hydraulic characteristic in function of the $\sigma$. In the case where the $c_{p, \min }$ value is near the trailing edge of the blade, which corresponds to low flow angle of attack, only a lift drop can be appear since the vapour pressure goes to limit the suction of the blade. In the other case where the $c_{p, \min }$ is reached near the leading edge, an increase of the lift is generated by the development of travelling bubble cavitation in the zone where the pressure is higher as the vapour pressure. The drop appears only when the vapour pressure is reached on the suction side.

\subsection{THE FRANCIS RUNNER}

Generally, the pressure distribution in a Francis runner at the best operating point corresponds to the first case that we described in the previous section. The flow angle of attack is low and the minimum pressure is localized on the outlet of the blade. The tests are conducted at this operating point for three different heads: $10 \mathrm{~m}, 15 \mathrm{~m}$ and $20 \mathrm{~m}$. The results of a flow computation in the case of a head of $15 \mathrm{~m}$ are presented on Figure 6 . One can observe on this figure that the minimum of the pressure is localized in the outlet of the blade for each computation mesh line except in the zone close to the runner band $(\mathrm{k}=19)$. Based on the conclusion of the previous described experiment, the efficiency must directly decrease with the cavitation coefficient. All the more so since the whirl has no effect at the best operating point. The cavitation coefficient usually used in hydraulic turbomachines is the Thoma number $\sigma=N P S E / E$.

The cavitation curves obtained are illustrated on Figure 7. The values of the efficiency are related to the cavitation free values. We can determine the value of $\sigma_{0}$ which is the highest value of the Thoma number where the efficiency is modified. This value is the same for all the tests with nuclei injection and is equal to 0.068 . The values of $\chi_{E}$ corresponding to this Thoma number are equal to $0.024,0.022$ and 0.02 for the three test heads. The com- 

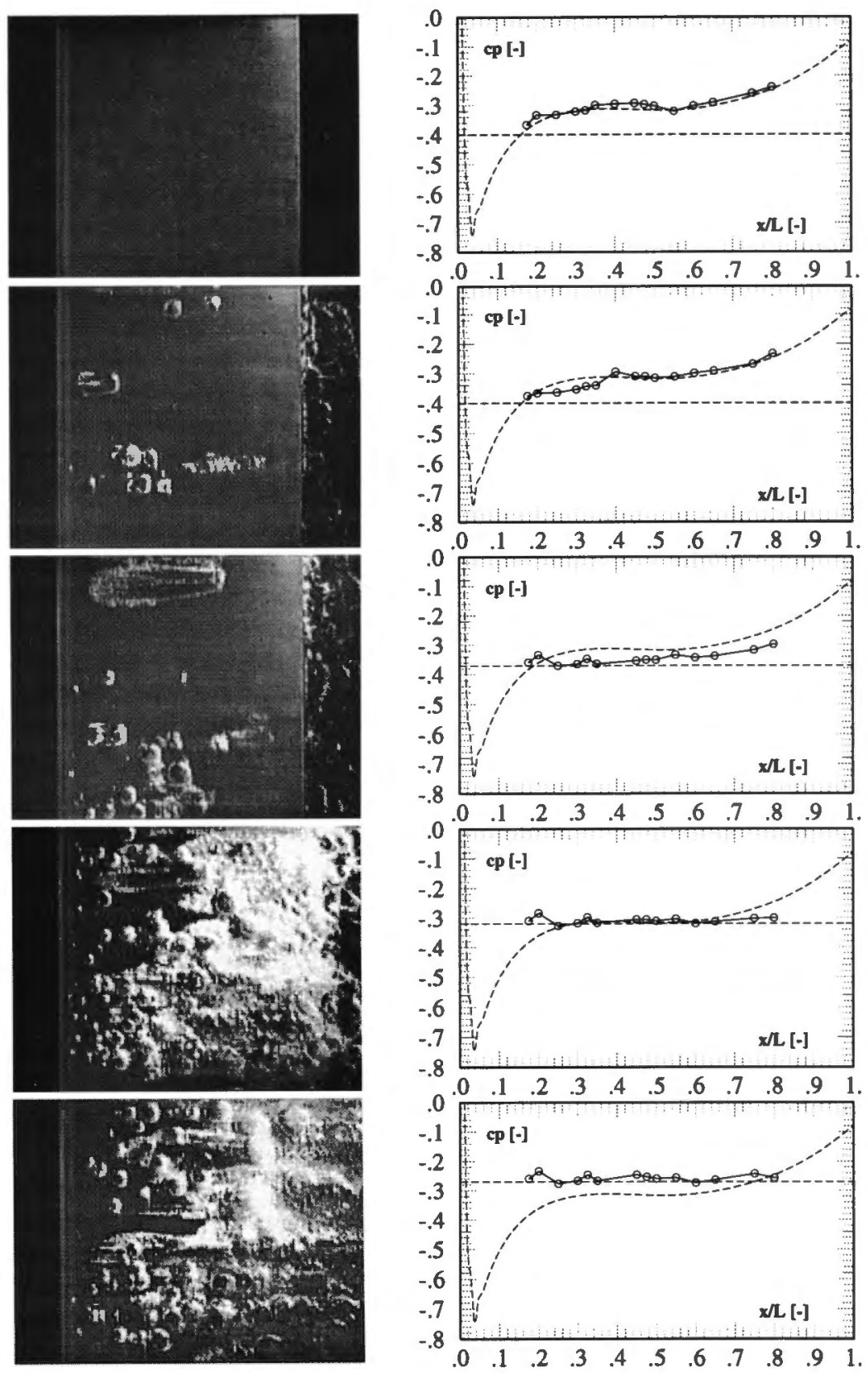

Figure 5. Measured and computed pressure distribution on the NACA 009 blade for the following values of $\sigma: 1.0,0.4,0.375,0.325,0.275$. (From top to bottom). The horizontal dashed line represents the $-\sigma$ value 


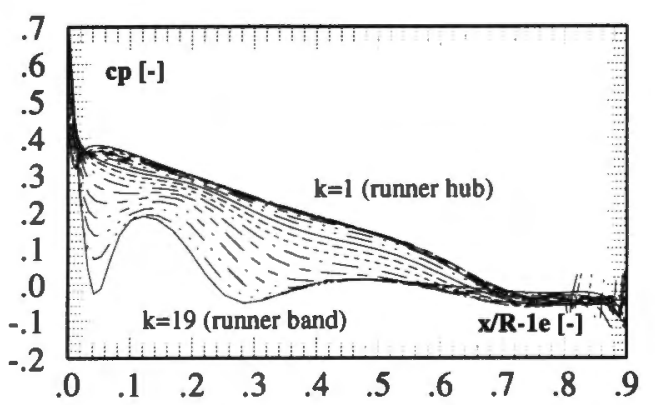

Figure 6. Results of a Navier-Stokes flow computation at the best operating point. The reference pressure is the computation domain outlet pressure. $\mathrm{H}=15 \mathrm{~m}$

parison with the pressure coefficient computed by the Navier-Stokes code shows us that the beginning of the efficiency drop appears when the all the values of the minimum pressure coefficient are lower as $-\chi_{E, 0}=-0.022$. At this state, it corresponds to an important development of the outlet cavitation. For the case corresponding to the computed one, the first bubbles are developed for a value of the Thoma number of 0.085 . Moreover, based on the computed results, we can determine the maximum value of the Thoma number where the vapour pressure is reached on the blade. The minimum pressure coefficient on the blade provides this value of $\sigma$ which is 0.11 . Thus, we have a difference of the $\sigma$ from 0.04 between the point where the vapour pressure is reached on the blades and the beginning of the efficiency drop. The development of travelling bubble cavitation must be then relatively established before the efficiency falls.

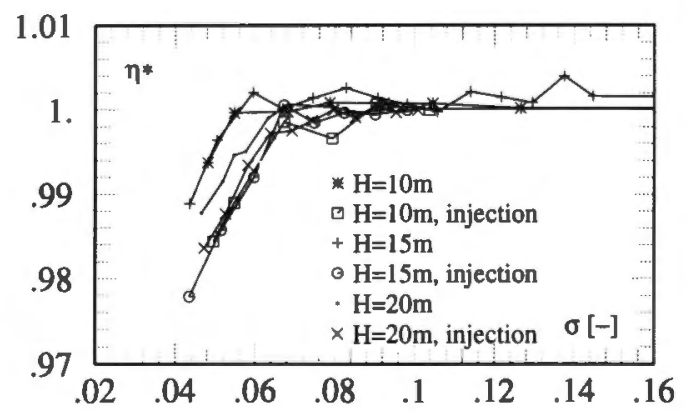

Figure 7. Normalized $\sigma-\eta$ curves for the Francis runner tests.

As in the case of the 2D blade, we can plot the results of the pressure measurements with the computed distribution at the corresponding computation mesh line. The Figure 8 presents these results where we can observe that the correspondence between the measurements and the computation is very good for the case without cavitation. For lower values of the Thoma 
number, the pressure distribution is modified by the development of travelling bubble cavitation. The different pressure measurements show us a decrease of the pressure until the vapour pressure value. The development of the bubbles is due to the upstream pressure drop localized close to the runner band. However, the main performance of the runner decrease since the development of the travelling bubble cavitation take effect mainly at the outlet of the blade.
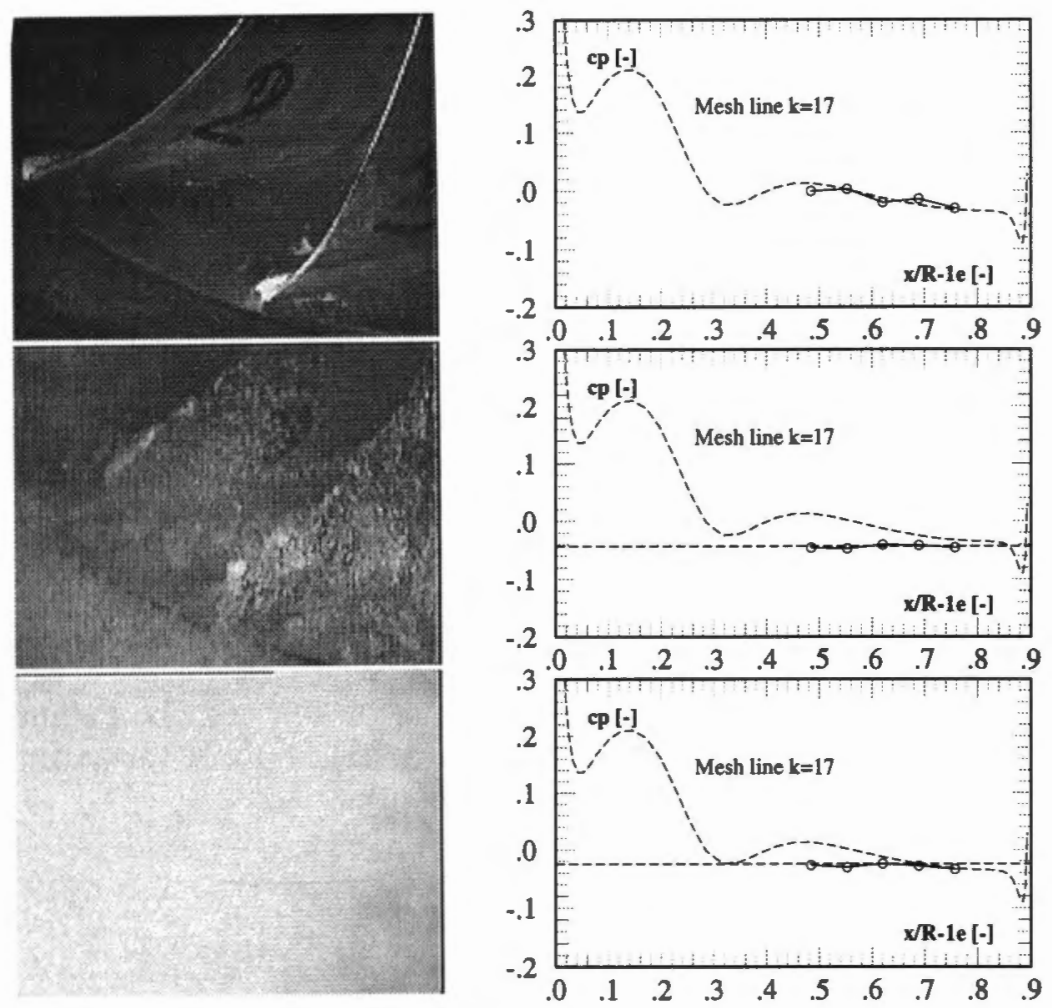

Figure 8. Measured and computed pressure distribution on the Francis runner blade for the following values of $\sigma: 0.43,0.07,0.06$. $\mathrm{H}=15 \mathrm{~m}$. (From top to bottom). The horizontal dashed line represents the $-\chi_{E}$ value.

\section{Conclusion}

Based on these two experiments, the effects of the travelling bubble cavitation on the turbomachines performances are better described. The efficiency modification appears mainly when the saturation of the cavitation is reached. The saturation causes a modification of the pressure distribution by limiting the pressure field to the vapour pressure value. The part of the machine where the shape of the bubbles stay spherical is not really influenced in term of performances. The acoustic emission caused by the 
bubbles growth or collapses is an other problem which justify the study of the acoustic pressure field around the active bubbles. However, in order to obtain a modeling of the pressure distribution modified by travelling bubble cavitation, it is necessary to determine the different zones of the blade where the bubbles are spherical, hemispherical and the cavitation saturation is reached. The size and the position of these different regions depends naturally to the cavitation nuclei concentration and to the operating point of the machine. This is the reason why the study of the bubbles evolution close to a wall in a variable pressure field is important for the characterization of the performances alteration in a hydraulic turbomachine. The way to this characterization in the case of the efficiency alteration by travelling bubble cavitation is certainly the study of a reliable model of the bubble evolution in a runner, and then the modification of its shape including the case of the saturation. The effects of the bubbles on a pressure field being now relatively known and described, this is the knowledge of the distribution of these different travelling bubble cavitation development on the runner that will be make possible a prediction of the efficiency drop in the Francis turbines due to this type of cavitation.

\section{Acknowledgment}

The authors are particularly grateful to the members of the IMHEF Cavitation research group and to its technical staff. This research is financially supported by the PSEL "Fonds Suisses pour Projets et Etudes de l'Economie Electrique".

\section{References}

1. Y. Kuhn de Chizelle et al. Observations and scaling of travelling bubble cavitation. J. Fluid. Mech., 293:99-126, 1995.

2. C. Brand et al. The imhef system for cavitation nuclei injection. Sao Paolo, 1992. AIRH.

3. Ch. Arn et al. Experimental and theoretical study of the 2d blade lift alteration by traveling bubble cavitation. ASME, August 1995.

4. F. Avellan et al. Theoretical and experimental study of the inlet and outlet cavitation in a model of francis turbine. pages 38-55, Stirling, August 1984.

5. F. Avellan et al. A new high speed cavitation tunnel for cavitation studies in hydraulic machinery. volume 57, pages 49-60. ASME, 1987.

6. F. Pereira et al. Dynamic calibration of transient sensors by spark generatd cavity. Symposium of Bubble Dynamic and Interface Phenomena, September 1993.

7. J.P. Franc et al. La Cavitation, Mécanismes physiques et aspect industriels. Presses Universitaires de Grenoble, 1995.

8. S. Kumar et al. A study of pressure pulses generated by traveling bubble cavitation. J. Fluid. Mech., 225:541-564, 1993.

9. B. Gindroz. Lois de Similitude dans les essais dans les Essais de Cavitation des Turbines Francis. PhD thesis, EPFL, 1991.

10. J.T. Daily R.T. Knapp and F.G. Hammit. Cavitation. Mac GRaw Hill, New York, 1970. 